\title{
Suicide risk and prevention in patients with schizophrenia
}

\author{
By Jill M. Harkavy-Friedman
}

The risk for suicidal behaviors in schizophrenia is high with rates estimated between $5.6 \%$ to $15 \%$ dying by suicide (Inskip et al., 1998; Palmer et al., 2005) and 20-40\% making suicide attempts (Caldwell \& Gottesman, 1990; Meltzer, 2002). The more we understand about risk factors and warning signs the better able we will be to prevent suicide. Overall, the research suggests that improved quality of life decreases the risk for suicide. With treatments and attention to those living with schizophrenia, more suicidal behavior can be prevented. Suicidal behavior is devastating to the family, community and society at large and deserves all of our efforts towards prevention. This paper reviews warning signs and factors found to be associated with increased risk for suicidal behavior. This review discusses attempted and completed suicide as well as general risk factors and risk factors associated specifically with schizophrenia.

\section{Risk factors and warning signs}

There are many risk factors for suicidal behavior and there is no one predictive risk profile that clinicians can rely on to identify the patient at imminent risk.

The process of managing suicidal behavior is ongoing throughout treatment and begins with a thorough evaluation of demographics, clinical and life experience factors. The general model for suicidal behavior proposed by Mann et al. (1999) suggests that diathesis variables lower the threshold for suicidal behavior and include genetic, clinical and historical factors such as mental illness, family history of suicide, aggression and abuse. They propose that more proximal trigger factors lead to the actual behavior and these factors include stressful events, an acute episode of psychosis or depression or substance use. This model predicts that some individuals are more vulnerable to suicidal behavior when faced with stressors. It is the confluence of risk factors that is helpful in identifying individuals at risk rather than one single factor or one predictive model. Some contributed factors are general to all populations and some specific to identified risk groups such as schizophrenia.

\section{Past suicidal behavior is the best predictor of suicide risk}

As in the general population, a history of a previous suicide attempt is the strongest predictor of future suicide attempts and death by suicide followed only by suicidal ideation (Roy, 1982; Drake et al., 1984; De Hert et al., 2001; Heila et al., 1997). A careful assessment of a patient's history of suicidal behavior will provide the most information regarding suicide risk although there are many who die by suicide as a result of their first suicidal act.

\section{Demographics of suicidal behavior}

There are demographic factors that describe higher risk groups in schizophrenia (see reviews by Hawton et al., 2005; Caldwell \& Gottesman, 1990; HarkavyFriedman \& Nelson, 1997). For example, males kill themselves more often than females yet the risk for females remains high unlike in the general population. For those with schizophrenia, individuals below age 45 and early in the course of illness are more likely to engage in suicidal behavior, both attempts and completed suicide, often before seeking psychiatric help and after the onset of psychosis. Nonetheless, the risk for suicide and suicide attempts extends throughout the lifespan in schizophrenia (Breier \& Astrachan, 1984; Harkavy-Friedman et al., 1999). Individuals who attempt and complete suicide have been shown to be more likely to live alone (Caldwell \& Gottesman, 1990; Harkavy-Friedman et al., 1999;

Hawton et al., 2005). The definition of employment is difficult and results related to employment status have been inconsistent. In general, demographic characteristics are a consideration but are not available for intervention.

\section{Clinical risk factors and warning signs}

Schizophrenia is a chronic mental illness that for some involves periods of acute exacerbation and periods of ongoing attenuated symptoms. Individuals with frequent relapses of psychotic symptoms have been found to be at higher risk (Hunt et al., 2006). Command auditory hallucinations can trigger suicidal behavior among those who are vulnerable and ought to be monitored closely. Being bothered by psychotic symptoms has been noted by patients as a precipitant for suicide attempts (Drake et al., 1984; Breier \& Astrachan, 1984; Harkavy-Friedman et al., 1999; Harkavy-Friedman et al., 2003). Depressed mood, hopelessness or a clinical depressive episode can trigger suicidal behavior although the rate of depression is high for nonsuicidal patients as well (Drake et al., 1984; Harkavy-Friedman et al., 2004; Hawton et al., 2005). For the vulnerable person, the risk increases during periods of increased depression especially when accompanied by hopelessness (Drake et al., 1984). Comorbid conditions such as alcohol and substance use disorders are frequently found to be associated with suicidal behavior as are changes in substance use behavior (Fowler et al., 1998; Verdoux et al., 1999; Hawton et al., 2005).

Anecdotally, for people with schizophrenia, a period of high risk is when a hospitalized person is told that they cannot return home after hospitalization. This may be because this often comes as a surprise to the patient and occurs at a time when the person is preparing for discharge and can come and go from the hospital openly. Restricting the patient to the hospital unit temporarily and discussing thoughts and feelings about discharge plans can help prevent suicidal behavior. The 3-6 months after hospital discharge is also a period of high risk (Caldwell \& Gottesman, 1990; Harkavy \& Nelson, 1997). The relationship between hospital discharge and suicidal behavior needs further study.

\section{Lifetime risk factors}

There has debate about the role of premorbid social functioning and insight into illness with respect to suicidal behavior in schizophrenia. Both poor premorbid social functioning and good premorbid social functioning have been implicated, probably because both reflect distinct paths to suicidal behavior. Those with poor premorbid functioning may lack problem-solving skills and therefore resort to suicidal behavior (Stephens et al., 1999). For those with good premorbid functioning, some investigators suggest a demoralization syndrome whereby typically wellfunctioning individuals become aware of their mental illness and feel demoralized 


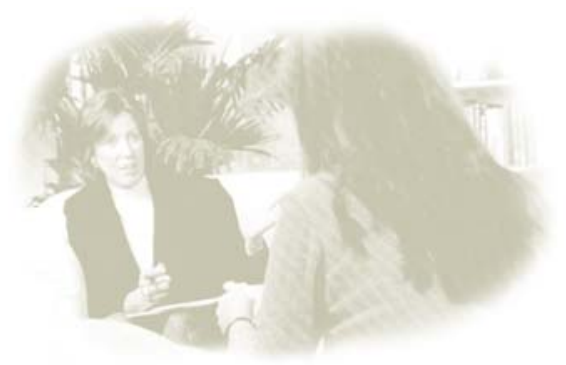

and resort to suicidal behavior (Drake et al., 1984). While both descriptions are plausible there are certainly individuals in both groups who find a way to live life with schizophrenia. A recent study found that good premorbid functioning was associated with increased depression but not suicide attempts (Restifo et al., 2009).

Other factors have also been implicated but there are no definitive findings yet. Poor engagement and adherence to treatment has been associated with suicidal behavior (Tiihonen et al., 2006; Hawton et al., 2005). Aggression and impulsiveness have also been implicated, particularly in light of the suggested relationships among these factors and serotonin (Brown \& Goodwin, 1986; Harkavy-Friedman et al., 1999).

\section{Protective factors}

There has been limited study of factors related to reducing suicidal behavior. The most frequently studied protective factor in schizophrenia is antipsychotic treatment and this has been shown to reduce the risk for suicidal behavior. Clozapine has been the most frequently studied and an anecdotal finding in their first study (Meltzer \& Okayli, 1995) led to The InterSept study (International Suicide Prevention Trial) (Meltzer et al., 2003), where suicidal behavior was studied more closely in suicide attempters. In that study, there was a lower rate of suicide attempts by those on clozapine and a lower rate of completed suicide by those on olanzapine. Other researchers have demonstrated that treatment with most antipsychotics can reduce the risk for suicidal behavior (Meltzer \& Boho, 2007; Haukka et al., 2008).

\section{Assessment of suicidal behavior}

It is critical to conduct a thorough evaluation of past and present suicidal behavior. Melle et al. (2006) demonstrated that individuals experience suicidal behavior early in the course of their illness and suggest that early intervention may be beneficial for reducing suicide risk.

It is important to be specific and ask the individual if they have ever thought about killing themselves and exactly what the thoughts have been. Questions about frequency, planfulness, persistence and method availability are essential. If the person has had suicidal ideas they will most likely feel some relief talking about them and if they never thought about suicide asking will not make them think about it. It is important to ask about past attempts by asking specifically whether or not they have ever tried to kill themselves or if they ever started to kill themselves and stopped at the last minute (aborted attempt) or were interrupted by someone else (interrupted attempt) (Posner et al., 2007). Intent is not necessarily represented by the lethality of an attempt so it is crucial to ask about the strength of the intent to die and the method used as well as how much medical damage was caused by the attempt. In order to try to prevent future attempts it is important to find out about precipitants and circumstances surrounding suicidal behavior including thoughts and behaviors before, during and after.

\section{Assessment of factors related to suicidal behavior}

Assess factors often associated with suicidal behavior such as clinical state, substance use, life experiences and problem solving strategies. With regard to psychotic behavior, assess the type, frequency and attitudes towards the symptoms as well as the strategies the person uses to manage the psychotic symptoms. Antipsychotic medications can reduce suicidal behavior (Meltzer \& Boho, 2007; Haukka et al., 2008) and therefore it is important to determine the medication type, dose, efficacy and side effects. With respect to depression, assessment of mood along with the presence of a major depressive episode ought to be assessed and treated with antidepressants or mood stabilizing medications and/or psychosocial therapies.

Assessment of risk factors and warning signs of suicidal behavior also includes asking about substance use including recent changes in patterns of use. Recent losses or other stresses may be important (Hawton et al., 2005; Harkavy-Friedman et al., 1999), especially taking into account available social supports and problem solving skills. Quality of life is important and therefore learning about the person's long-term treatment plan and hopes and aspirations for the future may help with the assessment of risk and the determination of possible interventions (Ponizovsky et al., 2003).

\section{Interventions for acute suicidal behavior}

Providing a safe haven is critical for the acutely suicidal patient with plan and intent. Hospitalization can provide a short-term safe haven but is not always necessary, depending upon available family and community supports. If the individual can be monitored continuously and all potential means of suicide are removed or restricted, the person may be safe and the suicidal crescendo may subside. For some, just sitting in an emergency department for a period of time can be enough. While protecting the patient, it is important to stabilize medications and symptoms and limit side effects. Mobilization of available social supports is essential. Sometimes a "plan for safety" for outpatients in general can help prevent acting on suicidal behavior (Stanley $\&$ Brown, 2008). A safety plan includes working with the individual regarding what they can do and whom they can contact should they feel actively suicidal in the future. This also enables supports to be aware, in advance, of how to help the person through a suicidal crisis. Deciding whether someone can remain outside the hospital with a safety plan or requires hospitalization depends on the above factors, i.e., intensity of suicidal behavior, means availability, presence of social supports.

\section{Longer-term intervention for suicidal behavior}

The person with schizophrenia who has suicidal behavior often experiences chronic or intermittent increases in suicidal behavior. Assisting the person through periods of high risk is critical. Researchers have shown that reducing patients' symptoms can reduce suicidal behavior. Psychological interventions that help the person to develop effective strategies for managing suicidal behavior and improving problem solving are helpful.

Antipsychotics, particularly secondgeneration antipsychotics, have been shown to reduce psychotic symptoms as well as suicidal behavior (Meltzer \& Boho, 2007; Haukka et al., 2008; Keck et al., 2000). It may be that reducing symptoms reduces stress and improves ability of function and problem solve, thereby reducing suicidal behavior. This requires further study. 
The InterSePT study (Meltzer et al., 2003) compared clozapine and olanzapine with respect to suicidal behavior among previous suicide attempters. Their findings suggested that clozapine might be superior to other medications while other investigators have demonstrated that treatment with antipsychotics in general reduces suicidal behavior (Haukka et al., 2008) again suggesting reducing symptoms of schizophrenia may reduce suicidal behavior. Though not well studied, treating comorbid depression with antidepressants or mood stabilizers is likely to help prevent suicidal behavior. Medication in conjunction with psychosocial interventions has been demonstrated as more effective than treatment as usual in the OPUS study of integrated treatment (Nordentoft et al., 2002).

Psychosocial interventions are being developed to assist in the treatment and prevention of suicidal behavior among individuals with schizophrenia. Bateman \& Hansen (2007) applied cognitive behavior therapy for preventing suicidal ideation in schizophrenia and found it effective. Other therapies are being developed but have not yet been tested.

\section{Conclusions}

Suicidal behavior is prevalent in schizophrenia. Getting through high-risk periods will help to prevent suicidal behavior. In patients with schizophrenia, continued monitoring of risk factors and precipitating circumstances is important. Intervening before the behavior gains force can help prevent suicide attempts. Psychopharmacological and social treatments for psychosis and depression are likely to reduce suicidal behavior along with interventions that provide alternate strategies for problem solving. When treating the patient with schizophrenia, especially the suicidal patient, don't give up but rather seek support and consultation if necessary. While not all suicides can be prevented, there are options for reducing suicide risk. Improving the quality of life for individuals with schizophrenia is likely to reduce the rate of suicide and other suicidal behaviors.

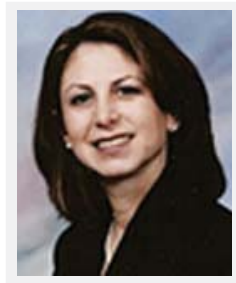

Jill M. Harkavy-Friedman, $\mathrm{PhD}$ is an Associate Professor of Clinical Psychology, in Psychiatry at Columbia University and a Research Scientist in the Department of Clinical Phenomenology at New York State Psychiatric Institute. She has significant expertise in the areas of psychiatric diagnosis and assessment as well as research methodology. Her primary areas of research are suicidal behavior in major mental illness, especially schizophrenia and the phenomenology of schizophrenia.

\section{References}

Bateman, K., \& Hansen, L. (2007). Cognitive behavior therapy reduces suicidal ideation in schizophrenia: Results from a randomized control trial. Suicide Life Threat Behav, 37(3): 284-290.

Breier, A., \& Astrachan, B.M. (1984). Characterization of schizophrenic patients who commit suicide. Am J Psychiatry, 141(2):206-9.

Brown, G.L., \& Goodwin, F.K. (1986). Cerebrospinal fluid correlates of suicide attempts and aggression. Ann N Y Acad Sci, 487:175-188

Caldwell, C.B., \& Gottesman, I.I. (1990). Schizophrenics kill themselves too: a review of risk factors for suicide. Schizophr.Bull, 16(4):571-589

De Hert, M., McKenzie, K., \& Peuskens, J. (2001). Risk factors for suicide in young people suffering from schizophrenia: a long-term follow-up study. Schizophr Res, 47(2-3):127-34.

Drake, R.E., Gates, C., Cotton, P.G., \& Whitaker, A. (1984). Suicide among schizophrenics. Who is at risk? J Nerv Ment Dis, 172(10):613-7.

Fowler, I.L., Carr, V.J., Carter, N.T., \& Lewin, T.J. (1998) Patterns of current and lifetime substance use in schizophrenia. Schizophr Bull, 24:443-455.

Harkavy-Friedman, J.M., Kimhy, D., Nelson, E.A., Venarde, D.F, Malaspina, D., \&, Mann, J.J. (2003). Suicide attempts in schizophrenia: The role of command auditory hallucinations for suicide. J Clin Psychi, 64(8): 871-874.

Harkavy-Friedman, J.M., \& Nelson, E. (1997). Management of the suicidal patient with schizophrenia. Psychiatr Clin North Am, 20(3):625-40

Harkavy-Friedman, J., Nelson, E., Amador, X., \& Mann, J.J. (1999). Aggression, impulsivity, suicidal behavior and clinical features in schizophrenia. Schiz Res (Abstract), 36:19.

Harkavy-Friedman, JM. Nelson, E.A., Venarde, D.F, \& Mann, J.J. (2004). Suicidal behavior in schizophrenia and schizoaffective disorder: Examining the role of depression. Suicide Life Threat Behav, 34(1), 66-76.

Harkavy-Friedman, J.M., Restifo, K., Malaspina, D., Kaufmann, C.A., Amador, X.F., Yale, S.A., et al. (1999). Suicidal behavior in schizophrenia: characteristics of individuals who had and had not attempted suicide. Am.J Psychiatry, 156(8):1276-1278.

Haukka, J., Tiihonen, J., Harkanen, T., \& Lonnqvist. J. (2008). Association between medication and risk of suicide, attempted suicide and death in nationwide cohort of suicidal patients with schizophrenia. Pharmacoepi Drug Safety, 17:686-696.

Hawton, K., Sutton, L., Haw, C., Sinclair, J., Deeks, JJ. (2005). Schizophrenia and suicide: A systematic review of risk factors. Brit J Psych, 187:9-20.

Heila, H., Isometsa, E.T., Henriksson, M.M., Heikkinen, M.E., Marttunen, M.J., \& Lonnqvist, J.K. (1997). Suicide and schizophrenia: A nationwide psychological autopsy study on age- and sex-specific clinical characteristics of 92 suicide victims with schizophrenia. Am J Psychiatry, 154:1235-1242.
Hunt, I.M., Kapur, N., Windfuhr, K., Robinson, J., Bickley, H., Flynn, S., et al. (2006). Suicide in schizophrenia: findings from a national clinical survey. J Psychiatr.Pract, $12(3): 139-147$

Inskip, H.M., Harris, E.C., \& Barraclough, B. (1998). Lifetime risk of suicide for affective disorder, alcoholism and schizophrenia. The Brit J Psychiatry, 172:35-37.

Keck, P.E., Jr., Strakowski, S.M., \& McElroy, S.L. (2000) The efficacy of atypical antipsychotics in the treatment of depressive symptoms, hostility, and suicidality in patients with schizophrenia. J Clin Psychiatry, 61 Suppl 3:4-9.

Mann, J.J., Waternaux, C., Haas, G.L., \& Malone, K. (1999). Toward a model of suicidal behavior in psychiatric patients. Am J Psychiatry, 156(2): 181-189.

Melle, I., Johannesen, J.O., Friis, S., Haahr, U., Joa, I., Larsen, T.K., Opjordsmoen, S., Rund, B.R., Simonsen, E., Vaglum, P., McGlashan, T., (2006). Early detection of the first episode of schizophrenia and suicidal behavior. Am J Psychiatry, 163: 800-804.

Meltzer, H.Y. (2002). Suicidality in schizophrenia: a review of the evidence for risk factors and treatment options. Curr Psychiatry Rep, 4(4):279-83.

Meltzer, H.Y., Alphsm L., Green, A.I., Altamura, A.C. Anand, R., Bertoldi, A., et al. (2003). Clozapine treatment for suicidality in schizophrenia: International Suicide Prevention Trial (InterSePT). Arch Gen Psychiatry, 60(1):82-91.

Meltzer, H.Y., \& Boho, W.V. (2007). Pharmacological Management of Suicide Risk in Schizophrenia. In R. Tatarelli, M. Pompili, \& P. Girardi (Eds). Suicide in Schizophrenia (pp. 275-302). New York: Nova Biomedical Books.

Meltzer H.Y., Okayli G. (1995). Reduction of suicidality during clozapine treatment of neuroleptic-resistant schizophrenia: impact on risk-benefit assessment. Am J Psychiatry;152:183-190.

Nordentoft, M., Jeppesen, P., Abel, M., Kassow, P., Petersen, L., Thorup, A., et al. 2002). OPUS study: suicidal behaviour, suicidal ideation and hopelessness among patients with first-episode psychosis. One-year follow-up of a randomised controlled trial. Br J Psychiatry Suppl, 43:98-106.

Palmer, B.A., Pankratz, V.S., \& Bostwick, J.M. (2005) The lifetime risk of suicide in schizophrenia: a reexamination. Arch Gen Psychiatry, 62(3):247-253. Ponizov sky., A.M., Grinshpoon, A., Levav, I., Ritsner, M.S. (2003). Comp Psychi, 44(6):442-447.

Posner, K., Oquendo, M.A., Gould, M., Stanley, B., \& Davies, M. (2007). Columbia Classification Algorithm for Suicide Assessment (C-CASA): Classification of suicide events in the PDA's pediatric suicidal risk analysis of antidepressants. Am J Psychiatry, 164: 1035-1043.

Restifo, K., Harkavy-Friedman, J., Shrout, P.E. (2009). Suicidal behavior in schizophrenia: A test of the demoralization hypothesis. J Ner Ment Dis, 197(3):147-153.

Roy A. (1982). Suicide in chronic schizophrenia. Br J Psychiatry, 141:171-7.

Stanley, B. \& Brown, G.K. (2008). Safety Plan Treatmen Manual to Reduce Suicide Risk: Veteran Version. Veterans' Administration.

Stephens, J.H., Richard, P., \& McHugh, P.R. (1999). Suicide in patients hospitalized for schizophrenia: 1913 1940. J Nerv Ment Dis, 187(1):10-4.

Tiihonen, J., Walhbeck, K., Lonnqvist, J., Klaukka, T. Ioannidis, J.P.A., Volavka, J., et al. (2006). Effectivenes of antipsychotic treatments in a nationwide cohort of patients in community care after first hospitalisation due to schizophrenia and schizoaffective disorder: observational follow-up study. BMJ, 333(7561):224.

Verdoux, H., Liraud, F., Gonzales, B., Assens, F., Abalan, F., \& van Os, J. (1999). Suicidality and substance misuse in first-admitted subjects with psychotic disorder. Acta Psychiatr Scand, 100:389-395. 